\section{G173(P) HOW SIGNIFICANT ARE ECGS AND 24 H HOLTER RECORDINGS IN CHILDREN PRESENTING WITH PALPITATIONS AND CHEST PAIN?}

${ }^{1} \mathrm{PS}$ Siddhi, ${ }^{2} \mathrm{M}$ Babu, ${ }^{2} \mathrm{M}$ Alsaffar. 'Neonatology, Birmingham Heartland Hospital, Birmingham, UK; ${ }^{2}$ Pediatrics, Mid Essex Hospital Trust, Broomfield, UK

\subsection{6/archdischild-2015-308599.168}

Aim We aim to identify the diagnostic significance of ECGs and $24 \mathrm{~h}$ Holter monitoring in children with palpitations and chest pain.

Methods We retrospectively analysed all children presenting with palpitations and chest pain to the emergency department and to out-patient clinics between 2008-2013. Data was collected through detailed review of case-notes. ECGs and Holter results were classified as abnormal if a significant cardiac arrhythmia was identified. Isolated ectopic beats (ventricular or supraventricular) were not considered as abnormal.

Children with previously diagnosed heart conditions were excluded.

Results Palpitation was the predominant symptom 71\% ( $\mathrm{n}=$ $42)$ with remainder $29 \%(\mathrm{n}=17)$ presenting with chest pain. $8 \%(\mathrm{n}=5)$ were infants, 46\%(27) were aged between 4-12 years and $46 \%(\mathrm{n}=27)$ were over 12 -years. $20 \%(\mathrm{n}=12)$ presented to the emergency department, whilst $80 \%(\mathrm{n}=47)$ were referred from the general practitioners.

ECG was performed in $97 \%(n=57)$ of cases during the initial assessment, of which $10 \%(n=6)$ were noted to be abnormal. 86\% ( $\mathrm{n}=49)$ of those who had ECGs underwent Holter monitor, of which, $18 \%(\mathrm{n}=9)$ were abnormal (the presenting history was palpitations in all these cases).

\begin{tabular}{lll}
\hline & ECG & $24 \mathrm{hrs} \mathrm{Holter}$ \\
\hline Sensitivity & $28 \%$ & $56 \%$ \\
Specificity & $97 \%$ & $100 \%$ \\
Positive predictive value & $83 \%$ & $100 \%$ \\
Negative predictive value & $74.5 \%$ & $82.5 \%$ \\
\hline
\end{tabular}

$43 \%$ ( $\mathrm{n}=18$ ) of the children with palpitations were diagnosed with a significant cardiac arrhythmia (12 with SVT, 3 with WPW, 3 with heart block). 57\%(n = 24) were non-significant sinus tachycardia.

$88 \%(n=15)$ of children with chest pain were diagnosed to have non-cardiac cause. However, 12\% ( $\mathrm{n}=2)$ were closely monitored due to a strong family history of cardiac arrhythmia.

Children with significant cardiac arrhythmias were referred to tertiary centre for further management.

Conclusion Palpitations and chest pain are not uncommon symptoms of presentation in the paediatric age group. The study demonstrates that ECGs are less sensitive compared to the 24hour Holter, but in combination they are useful non-invasive diagnostic tools. Chest pain in children is probably of little concern as compared to palpitations and further investigations should only be requested if clinically indicated.

\section{G174(P) INTRODUCING PULSE OXIMETRY AS A SCREEN FOR CRITICAL CONGENITAL HEART DEFECTS IN THE DISTRICT GENERAL HOSPITAL SETTING}

MR Abumehdi, U Srirambhatia, K Jada, C Cane, T Wickham. Neonatology, Barnet General Hospital, Barnet, UK

10.1136/archdischild-2015-308599.169
Aims Critical congenital heart defects (CCHD's) are associated with significant mortality and morbidity. Early diagnosis and treatment could reduce this significantly. Currently there is a diagnostic gap in identifying CCHDs even after prenatal screening and postnatal examination. Pulse Oximetry as part of newborn examination is increasingly being used to minimise this gap. There is a growing body of evidence to suggest that it is a valid screening tool for identifying CCHDs, which is reflected by its uptake in many neonatal units.

Our aim was to demonstrate the feasibility of implementing pulse oximetry screening in a busy DGH setting and outline the financial and practical considerations.

Methods All newborn examinations performed by paediatricians in July/August 2014 included pulse oximetry screening. A portable pulse-oximeter with disposable wraps was used to measure pre-ductal and post-ductal saturations. Outcomes were divided into three groups. Those with both readings $\geq 95 \%$ were deemed negative. Those with either reading between $90-94 \%$, or $>2 \%$ difference of saturations were deemed as a borderline positive test. These required re-measuring after $2 \mathrm{~h}$ if the child remained asymptomatic. Three borderline positive tests, or a positive test (either saturation $<90 \%$ or a symptomatic child) required comprehensive assessment and echocardiography where clinically indicated. Exclusion criteria were: Midwife performed newborn examinations, pre-term infants ( $<37$ weeks gestation), infants admitted to the neonatal intensive care ward.

Results 170 infants had their pre and post ductal saturations measured over a 1 -month period. Average time to testing was $38.6 \pm 27.5 \mathrm{~h}$. The average duration of the test was 3.98 $( \pm 3.41)$ minutes. All tests done over the trial period were negative. There was no increased burden on the echocardiography workload or SCBU beds. Feedback from doctors, nurses and parents was positive.

Conclusion Implementing pulse oximetry screening for CCHD is a simple and cost affective strategy, without significantly increasing the time taken for newborn examination. It has a reassuring effect on doctors performing newborn examination. As reported in other studies, delaying the screening until after $24 \mathrm{~h}$ maximises the specificity, which may explain all tests being negative. There was no increase in demand for echocardiograms or admissions onto the neonatal unit.

\section{Paediatric Education Special Interest Group}

\section{G175 "LEARNING TOGETHER": DO INTEGRATED CHILD HEALTH CLINICS EDUCATE AND IMPROVE OUTCOMES?}

${ }^{1} \mathrm{CP}$ Macaulay, ${ }^{2}$ E Sherwood, ${ }^{3} \mathrm{~W}$ Riches, ${ }^{3} \mathrm{M}$ Lakahanpaul, ${ }^{4} \mathrm{~J}$ Spicer. ${ }^{1}$ Paediatrics, Evelina London Children's Hospital, London, UK; ${ }^{2}$ Paediatric Emergency Department, Kings College Hospital, London, UK; ${ }^{3}$ Children's and Young People Programme, UCL Partners London, UK; ${ }^{4}$ Primary Care Education, Health Education South London, London, UK

\subsection{6/archdischild-2015-308599.170}

Background A significant number of children seen within secondary care, both in emergency departments, and also in outpatients, could be seen within a primary care setting (Saxena 2009, Milne 2010). Currently general paediatric postgraduate training programmes are focused on training paediatricians almost exclusively in hospital-based systems.

RCGP data (2012) reports that fewer than half of general practitioners (GPs) in training currently have an opportunity to gain experience of acute childhood illness in a specialist-based 